\title{
Development and Implementation of Electronic Disease Early Warning Systems for Optimal Disease Surveillance and Response during Humanitarian Crisis and Ebola Outbreak in Yemen, Somalia, Liberia and Pakistan
}

\section{Kamran Ahmed ${ }^{1}$, Muhammad Arish Salam Bukhari ${ }^{2}$, Mohammad Dauod Altaf ${ }^{3}$, Peter Clement Lugala $^{4}$, Ghulam Rabani Popal ${ }^{5}$, Alaa Abouzeid ${ }^{6}$, Margaret Lamunu ${ }^{7}$}

1. World Health Organization, Ex. eDEWS Project Team Lead for Yemen, Pakistan, Somalia and Liberia

2. World Health Organization, Technical Officer Information Systems, Regional Office for Africa (AFRO), Brazzaville

3. World Health Organization, Team Leader WHO Health Emergencies, Country Office, Afghanistan

4. World Health Organization, Officer in charge / acting WHO Representative, Country Office, Nigeria

5. World Health Organization, Senior Advisor to the Regional Director, Regional Office for the Eastern Mediterranean (EMRO), Cairo

6. World Health Organization, Emergency Operation Manager / Team Lead Operational Partnerships, Regional Office for the Eastern Mediterranean (EMRO), Cairo

7. World Health Organization, Health Emergency Officer, Headquarter, Geneva

\begin{abstract}
Objective: To share lessons learned with experience in concept development of electronic disease early warning system (eDEWS) as a standardized informatic tool for optimal disease surveillance for early warning and response Network (EWARN) during humanitarian crisis.

Methods: We did literature search, review and analysis to document system attributes of existing electronic tools being used for disease surveillance, early warning and health management information system (HMIS). We generated baseline information and conducted multiple planning sessions with stakeholders for EWARN system requirement elicitation and validation to inform concept development of standardized electronic tool.

Results: We identified 98 electronic health projects, classified 22 projects under 'Disease and epidemic outbreak surveillance' theme, whereas only four electronic tools met our selection criteria and were reported to be implemented in humanitarian settings complimentary to EWARN. Baseline information was obtained to guide work on requirement gathering and analysis process, and development of concept for a standardized electronic tool for EWARN.

Discussion: The eDEWS was enhanced with an objective to develop standardize electronic tools and data collection procedures to monitor diseases and health events for alert detection in global
\end{abstract}


humanitarian settings. The enhanced system could be harnessed as a powerful tool by outbreak response teams in getting vital epidemiological information for appropriate and timely response during emergencies.

Conclusion: eDEWS experiences in Yemen, Somalia, Liberia and Pakistan offers an opportunity to learn and apply lessons to improve future health informatics initiatives or adapt eDEWS as a feasible standardized approach to enhance EWARN implementation during humanitarian crisis, and potential integration into routine surveillance systems.

Keywords: Electronic Data Collection, Alert Notifications, Disease Early Warning, Electronic Tools, eDEWS, Humanitarian Crisis.

Abbreviations: Data summarization technique (DST), electronic disease early warning system (eDEWS), electronic integrated disease early warning system (eIDEWS), early warning and response network (EWARN), early warning and response system (EWARS), early warning and response (EWAR), global system for mobile communications (GSM), health management information system (HMIS), internally displaced populations (IDPs), information technology (IT), short messaging service (SMS), World Health Organization (WHO)

Corresponding Author: Kamran Ahmed - drkamranrajput@gmail.com

DOI: 10.5210/ojphi.v11i2.10157

Copyright (C2019 the author(s)

This is an Open Access article. Authors own copyright of their articles appearing in the Online Journal of Public Health Informatics. Readers may copy articles without permission of the copyright owner(s), as long as the author and OJPHI are acknowledged in the copy and the copy is used for educational, not-for-profit purposes.

\section{Introduction}

Displacement of population due to conflict and natural disasters, inadequate shelter, insufficient and unsafe water, and inadequate sanitation poses significant risk factors associated with potentially life-threatening infectious disease outbreaks. The extensive population movements during humanitarian crisis exacerbate the circumstances with significant numbers of internally displaced populations (IDPs), returnees and refugees congregating in urban centres and the outskirts where basic service provision and infrastructures are unable to absorb the additional burden, and services are overwhelmed or simply not available to address mounting needs [1,2]. Such poor conditions frequently result in increased morbidity and mortality during the crises, particularly in countries with poor disease surveillance mechanisms [3]. The routine public health surveillance systems of many low- or middle-income countries may be underperforming or nonexistent and may be disrupted during the humanitarian emergencies. The World Health Organization (WHO) recommends implementation of EWARN within two weeks of onset of an acute event as one of the priority interventions to mitigate the negative health consequences resulting from the acute emergency or humanitarian event. The EWARN system facilitates collection of essential, minimal data on prioritized epidemic prone or selected diseases with significant public health consequences, where rapid analysis of trends for outbreak or event 
detection for prompt response and intervention is instrumental for mitigating the potential high morbidity and mortality that may be attributed to the even. The implementation of EWARN in emergencies or for disaster response is recommended for emergency phase of the response and should be discontinued in the event of implementation or activation of routine disease surveillance system as the affected populations recovers from the emergency and /or disaster effects [4,5].

EWARN has been implemented as adjunct to the routine public health surveillance systems as a part of an earlier efforts to strengthen surveillance and response during the humanitarian emergencies, but it continued to use traditional paper recording and reporting tools to manage epidemiological data in several low- and middle-income countries such as Yemen, Somalia, Liberia, Iraq and Pakistan [6]. This has posed unique challenge to achieve EWARN objectives, consequently resulting in worsening of the health status of affected communities and populations at risk, when outbreaks of epidemic prone diseases remained undetected or detected too late to allow for effective public health response $[4,6,7]$.

Recent advances in public health informatics have however, led to the development and implementation of various electronic tools for different settings and context that facilitate real time reporting, transmission and processing of epidemiological data for timely detection, verification and prompt action. The electronic data capture using these tools have been aided using electronic devices such as mobile phones, tablet devices and other touch screen interfaces in certain settings utilizing existing IT infrastructure and communication means [8,9]. The electronic tools developed so far for disease surveillance or for similar purposes, are either in use or at various stages of development or evolution and with varying capabilities. Such disparate electronic tools with different functionalities and specificities, have been developed independently on different platforms using different architectures. Consequently, there is duplication of efforts, loss of time, lack of standardization and harmonization, leading to inefficient use of limited resources, including time. Also, disparate tools receive less feedback for improvement than if a single standard tool were recommended and broadly adopted [6,10-14]. Furthermore, published data on such tools is very limited [6,11,13,15-17].

While multiple challenges have been reported with implementation of EWARN in humanitarian setting, the need for standardization of electronic tools for data collection, analysis and reporting for use in humanitarian settings when existing public health surveillance systems are overburdened, underperforming or non-existent at all remains a critical necessity for public health workers in such settings. Equally, important is the need to address concerns over data privacy, protection, ownership and trust issues among stakeholders including governments of affected countries $[4,6]$.

This paper presents our efforts in conceptualizing, development and use of a standardized online database system with web-based and mobile/smart phone data collection application package, called 'electronic disease early warning system (eDEWS), and sharing lessons learned during its implementation in various context that include during acute, protracted and post emergency phases in humanitarian settings of Yemen (civil unrest), Somalia (civil unrest), Liberia (Ebola outbreak), Iraq (civil unrest) and Pakistan (Flood emergency) [13,15-18]. 


\section{Methods}

Various electronic tools with different functionalities and specificities (and using different architectures) have been developed and used for the purposes of EWARN implementation in humanitarian settings but published data on such tools is very limited $[6,11,13,15-17]$. To increase our understanding and knowledge on existing electronic tools, we did a preliminary work to analyze existing tools for system attributes, processes and lessons learned and later developed requirement specification document during planning sessions with stakeholders for the development of an online disease early warning database and electronic tools as a standardized data collection, analysis and reporting approach. The aim of development of standardized tool and data collection procedure was to enhance implementation of EWARN during humanitarian crisis with a potential to integrate into routine surveillance systems or fill gaps where no routine surveillance system exists.

We generated baseline information on existing electronic tools implemented for EWARN or similar purposes in various locations, as part of the generation and documentation of requirements for the development of standardized electronic tools and online database system. For this purpose, we did literature search and performed review and analysis to document functionalities and specifications of different electronic tools being used for various needs in health, including for early warning, disease surveillance and health management information systems. We used snowball literature search method to search available resources on existing electronic healthcare projects in PubMed/MEDLINE, Science Citation Index (Web of Science) and Google Scholar databases, including WHO library database for publicly available contents and writing to professional contacts working in humanitarian settings [19]. We used the keywords "Electronic Data Collection Tools" AND ("Early Warning" OR "Alert notification" OR "eHealth" OR "eSurveillance" OR "mHealth" OR “epidemics" OR "emergencies" OR "disease surveillance” OR "Outbreak response" OR "humanitarian response" OR "low income" OR "middle income"). The results of the above search were reviewed to identify tools meeting selection criteria and removed articles with no accessible abstracts and/or full-text versions.

We reviewed 100 articles and generated list of seven application areas in public health practice outlined below, where electronic tools have been developed and used to support various healthcare interventions:

1. Disease and epidemic outbreak surveillance

2. Health education and awareness

3. Health financing

4. Health management and information system

5. Health human resource (HRM) mLearning

6. Patient monitoring and support

7. Point of care, support and diagnostics

At least $23 \%$ of listed electronic tools were classified under 'Disease and epidemic outbreak surveillance' theme. Next, we filtered the list to select relevant electronic tools used in 
humanitarian emergency context and for more focused review and analysis using the following criteria;

1) Surveillance of infectious disease epidemics

2) Complementing routine surveillance systems

3) Outbreak detection and alerting

4) Use in humanitarian response

To obtain baseline information to guide efforts in gathering and analysis of requirements, process, and development of a standardized electronic tool to enhance implementation of EWARN in emergencies, we then performed an objective analysis and evaluation of specifications, functionalities, outputs, software details and positive attributes of individual tools, including gap analysis and challenges. $(6,11,14,16)$.

\section{Results}

We identified 98 health projects that have developed and implemented electronic tools in order to improve overall health care capacity and capabilities in various developing countries since 2000. Table 1 shows distribution of identified tools classified under different application areas for electronic health projects in public health practice.

Table 1: Classification of electronic tools being used in public health settings $(\mathrm{N}=98)$

\begin{tabular}{ll}
\hline Application Areas & Number of Initiatives \\
\hline Disease and epidemic outbreak surveillance & 22 \\
\hline Health education and awareness & 17 \\
\hline Health financing & 3 \\
\hline Health management and information system & 15 \\
\hline Health human resource (HRM) mLearning & 3 \\
\hline Patient monitoring and support & 24 \\
\hline Point of care, support and diagnostics & 14 \\
\hline Total articles selected & 98 \\
\hline
\end{tabular}

Following is the list of selected four electronic tools chosen from 'Disease and epidemic outbreak surveillance' theme based on selection criteria for more focused review and analysis to obtain baseline information:

1) District Health Information System (DHIS2)

2) Surveillance Post Extreme Emergencies and Disasters - Philippines (SPEED)

3) Electronic Disease Surveillance System for CISDCP - China

4) Electronic Disease Early Warning System - Pakistan 
The specification requirement document was generated as a result of review of these four tools and used in concept development process of eDEWS as a standardized tool to enhance EWARN implementation. The eDEWS was then developed and implemented during humanitarian crisis in Pakistan, Yemen, Somalia and Liberia, and adapted for EWARN implementation in Iraq.

The eDEWS attributes and lessons learned with experience in eDEWS implementation in these humanitarian settings are discussed in the next sections.

\section{eDEWS application concept, exit strategy and integration into routine surveillance system}

The eDEWS is an initiative that was designed for the first time during the flood emergencies in Pakistan in 2010 as a result of recommendations from external evaluation of disease early warning system [7,20]. eDEWS was developed with an objective to collect data using electronic data collection procedure to monitor diseases and health trends and could be harnessed as a powerful tool by health emergency managers in getting vital epidemiological information for appropriate and timely response during emergencies and disasters. eDEWS uses online database system complimented with web-based and mobile/smart phone-based user interfaces that allow communities, field epidemiologists and health workers in health facilities or selected sentinel sites to send daily or weekly disease data to the central database system through secure and timely electronic transmission for timely analysis. Built-in short messaging service (SMS) alert notification module in eDEWS analyzes data automatically and generates automatic SMS alert notifications to outbreak response teams for immediate verification and response. To generate reports and visualizations, health staff also validates data using advanced validation module within user interfaces before the submitted data is analyzed automatically to generate tables, graphs, epidemiological bulletins/reports and interactive data visualizations on dashboard including LIVE maps based on time, place and person. The electronic data collection application for eDEWS is developed considering compatibility with all operating systems include eDEWS mobile app for android/ios (with both online and offline data collection modes), desktop app for use in windows/mac/linux operating systems, satellite app and SMS or global system for mobile communications (GSM) approach for settings where GSM technology exist without mobile internet. All these apps are optional to be selected for specific context during the eDEWS implementation and included in one package that allows higher flexibility for customization of eDEWS and launching this application considering budget constraints. These optional features can be activated anytime based on needs and resources availability during the humanitarian crisis or while integrating eDEWS into routine surveillance system, figure 1. 


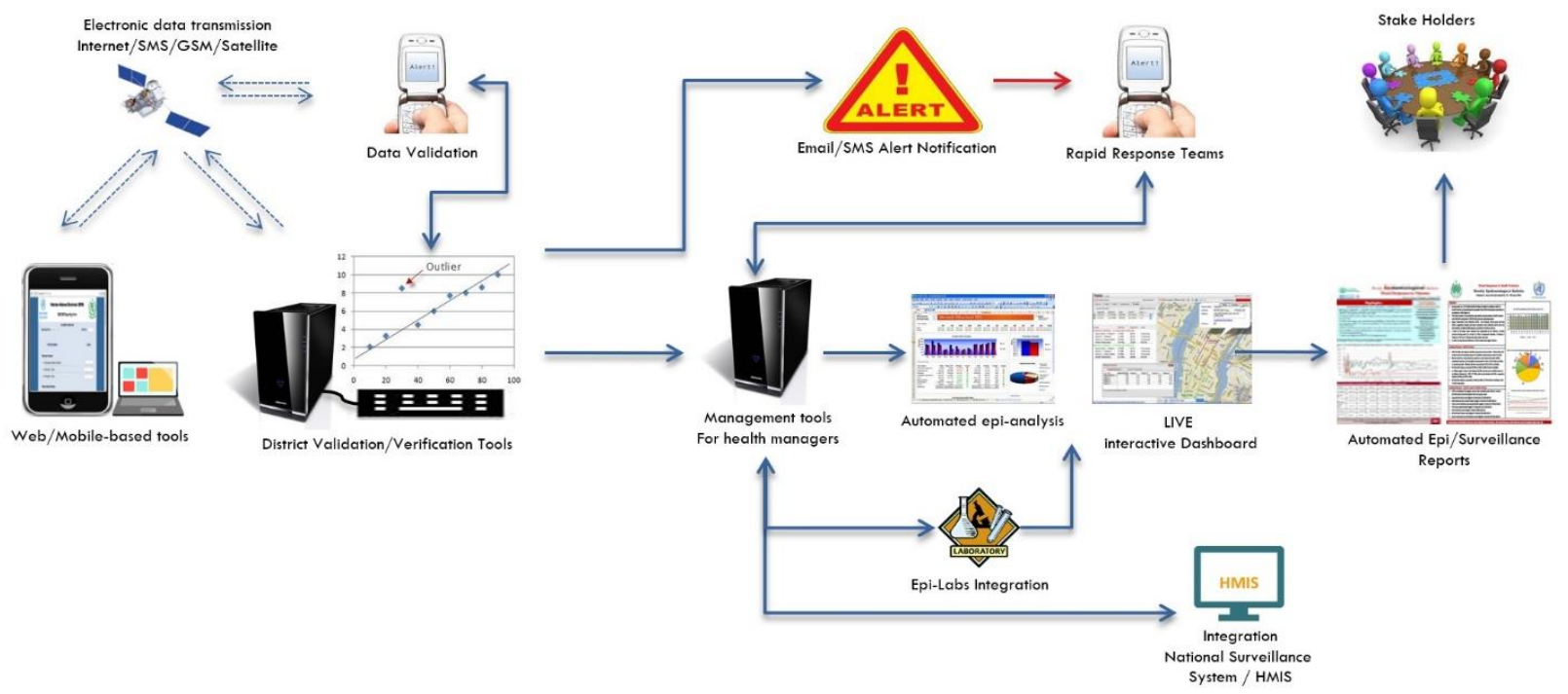

Figure 1: Technical diagram showing eDEWS workflow and integration with lab-information systems, national surveillance system and HMIS

The eDEWS has proven as an effective in its core functions of alert detection and early outbreak containment and able to transmit data from any geographical location with or without internet. Satellite apps don't require expensive satellite phones or systems but can upgrade smart phones to satellite data collection devices using cost effective boosters.

\section{Data Summarization Tool}

Data summarization technique (DST) is an innovative and low-cost simple analytical method that was developed to assess the disease situation for early detection of epidemics while launching implementation of eDEWS application during humanitarian crises in resource poor countries. This approach can easily bridge the gap between onset of emergency, planning, configuration and development phase for eDEWS implementation and final launch of eDEWS application as a standardized data collection and analysis procedure that takes two weeks to one month on average. The DST approach is easily implementable in practice and has been developed on advanced time efficient Ms-Excel pivot table technique that requires basic Excel skills at end user level and is customizable for better integration with other databases (example Epi Info \& Access) of vertical health programs to generate semi-automated epidemiological reports on timely and ad-hoc basis, an important public health informatics approach to perform well during emergencies. Local capacity is easy to build to utilize DST tools for complete data analysis using simulation exercises where basic Ms.Excel knowledge was considered essential for health staff participation in trainings. DST approach also improved timeliness of sharing of information on epidemiological trends and feedback back to stake holders and reporting facilities from weeks with paper recording system to few hours with DST in humanitarian settings of Yemen, Somalia and Pakistan [16]. 


\section{eDEWS attributes developed and implemented during humanitarian crisis}

Table 2 shows the summary of attributes developed and configured in developed eDEWS based on requirements specifications gathered and analyzed using baseline information from objective analysis and evaluation of selected electronic tools, and later used in various scenarios considering local context, surveillance needs, availability of resources and political considerations in humanitarian settings. The eDEWS application was fully implemented by WHO during the emergencies in Yemen, Somalia, Liberia and Pakistan and later adapted eDEWS for development and configuration of similar application to compliment EWARN implementation in Iraq in 2015 [15]. 
Table 2. Summary of system attributes of eDEWS implemented for EWARN enhancement in humanitarian settings of Yemen, Somalia, Liberia, Pakistan and Iraq*.

\begin{tabular}{|c|c|c|c|c|c|}
\hline $\begin{array}{l}\text { Features and } \\
\text { Functionalities }\end{array}$ & $\begin{array}{l}\text { Pakistan (2011- } \\
\text { 12) }\end{array}$ & $\begin{array}{l}\text { Yemen }(2013- \\
\text { present })\end{array}$ & $\begin{array}{l}\text { Somalia (2013- } \\
\text { 2015) }\end{array}$ & Liberia (2015-2016) & $\operatorname{Iraq}(2015) *$ \\
\hline $\begin{array}{l}\text { Reporting user } \\
\text { interfaces }\end{array}$ & $\begin{array}{l}\text { Web, Smart } \\
\text { Phone }\end{array}$ & Web, Smart Phone & $\begin{array}{l}\text { Web, Smart } \\
\text { Phone }\end{array}$ & $\begin{array}{l}\text { Web, Smart Phone, } \\
\text { SMS and Satellite }\end{array}$ & $\begin{array}{l}\text { Web, Smart } \\
\text { Phone }\end{array}$ \\
\hline Mobile app modes & $\begin{array}{l}\text { Internet, Offline } \\
\text { Sync }\end{array}$ & $\begin{array}{l}\text { Internet, Offline } \\
\text { Sync }\end{array}$ & $\begin{array}{l}\text { Internet, Offline } \\
\text { Sync }\end{array}$ & $\begin{array}{l}\text { Package (Internet, } \\
\text { Offline Sync, } \\
\text { SMS/GSM, Satellite) }\end{array}$ & $\begin{array}{l}\text { Internet, Offline } \\
\text { Sync }\end{array}$ \\
\hline $\begin{array}{l}\text { Automated } \\
\text { epidemiological } \\
\text { analysis }\end{array}$ & DST, Web-based & DST, Web-based & DST, Web-based & Web-based & Web-based \\
\hline $\begin{array}{l}\text { Alert notification } \\
\text { module }\end{array}$ & SMS notification & SMS notification & SMS notification & $\begin{array}{l}\text { SMS/Email } \\
\text { notification }\end{array}$ & $\begin{array}{l}\text { SMS/Email } \\
\text { notification }\end{array}$ \\
\hline Data transmission & $\begin{array}{l}\text { Internet, } \\
\text { SMS/GSM }\end{array}$ & $\begin{array}{l}\text { Internet, } \\
\text { SMS/GSM }\end{array}$ & $\begin{array}{l}\text { Internet, } \\
\text { SMS/GSM }\end{array}$ & $\begin{array}{l}\text { Internet, SMS/GSM, } \\
\text { Satellite }\end{array}$ & Internet, SMS \\
\hline $\begin{array}{l}\text { Data Integrity and } \\
\text { protection }\end{array}$ & Yes & Yes & Yes & Yes & Yes \\
\hline $\begin{array}{l}\text { Database and Apps } \\
\text { used }\end{array}$ & Open source & Open source & Open source & Open source & N/A \\
\hline $\begin{array}{l}\text { Form customization } \\
\text { feature }\end{array}$ & Yes & Yes & Yes & Yes & No \\
\hline $\begin{array}{l}\text { App for } \\
\text { communication with } \\
\text { end users }\end{array}$ & Yes & Yes & Yes & Yes & No \\
\hline Data visualization & LIVE interactive & LIVE interactive & LIVE interactive & LIVE interactive & LIVE interactive \\
\hline
\end{tabular}




\begin{tabular}{|c|c|c|c|c|c|}
\hline $\begin{array}{l}\text { Epidemiological } \\
\text { Bulletin }\end{array}$ & $\begin{array}{l}\text { Online, } \\
\text { Downloadable in } \\
\text { PDF }\end{array}$ & $\begin{array}{l}\text { Online, } \\
\text { Downloadable in } \\
\text { PDF }\end{array}$ & $\begin{array}{l}\text { Online, } \\
\text { Downloadable in } \\
\text { PDF }\end{array}$ & $\begin{array}{l}\text { Online, } \\
\text { Downloadable in PDF }\end{array}$ & N/A \\
\hline Reporting frequency & Daily, Weekly & Daily, Weekly & Daily, Weekly & Daily, Weekly & Daily, Weekly \\
\hline Current status & Exit & $\begin{array}{l}\text { Integrated into } \\
\text { routine health } \\
\text { system }\end{array}$ & Exit & Exit & N/A \\
\hline Data storage & $\begin{array}{l}\text { Local physical } \\
\text { server }\end{array}$ & $\begin{array}{l}\text { Local physical } \\
\text { server }\end{array}$ & $\begin{array}{l}\text { Local physical } \\
\text { server }\end{array}$ & Local physical server & N/A \\
\hline Import / Export data & $\begin{array}{l}\text { Yes (Excel, CSV, } \\
\text { XML) }\end{array}$ & $\begin{array}{l}\text { Yes (Excel, CSV, } \\
\text { XML) }\end{array}$ & $\begin{array}{l}\text { Yes (Excel, CSV, } \\
\text { XML) }\end{array}$ & $\begin{array}{l}\text { Yes (Excel, CSV, } \\
\text { XML) }\end{array}$ & N/A \\
\hline $\begin{array}{l}\text { Built-in data } \\
\text { validation \& } \\
\text { verification modes }\end{array}$ & Yes & Yes & Yes & Yes & Yes \\
\hline GIS mapping & $\begin{array}{l}\text { Interactive and } \\
\text { Realtime }\end{array}$ & $\begin{array}{l}\text { Interactive and } \\
\text { Realtime }\end{array}$ & $\begin{array}{l}\text { Interactive and } \\
\text { Realtime }\end{array}$ & $\begin{array}{l}\text { Interactive and } \\
\text { Realtime }\end{array}$ & $\begin{array}{l}\text { Interactive and } \\
\text { Realtime }\end{array}$ \\
\hline $\begin{array}{l}\text { Access and } \\
\text { permission }\end{array}$ & $\begin{array}{l}\text { Granular } \\
\text { permissions }\end{array}$ & $\begin{array}{l}\text { Granular } \\
\text { permissions }\end{array}$ & $\begin{array}{l}\text { Granular } \\
\text { permissions }\end{array}$ & Granular permissions & N/A \\
\hline $\begin{array}{l}\text { Lab support } \\
\text { integration }\end{array}$ & Yes & Yes & No & No & No \\
\hline $\begin{array}{l}\text { Outbreak } \\
\text { investigation module }\end{array}$ & Yes & Yes & Yes & Yes & No \\
\hline $\begin{array}{l}\text { Application } \\
\text { Programming } \\
\text { Interface (API) }\end{array}$ & Yes & No & No & Yes & N/A \\
\hline
\end{tabular}




\begin{tabular}{llllll}
\hline & & & & & \\
\hline $\begin{array}{l}\text { Reference guides and } \\
\text { FAQs }\end{array}$ & Yes & Yes & Yes & N/A \\
\hline $\begin{array}{l}\text { Integration with } \\
\text { routine surveillance } \\
\text { systems }\end{array}$ & Yes & No & No & N/A \\
$\begin{array}{l}\text { Embedded technical } \\
\text { guides }\end{array}$ & Yes & No & No & Yes & N/A
\end{tabular}

* Lack of access to the adapted eDEWS electronic tools and its documentation for EWARN in Iraq precluded meaningful comparison between eDEWS adaptation in Iraq with eDEWS implementation for EWARN in other humanitarian settings. 


\section{Challenges in setting up of eDEWS in humanitarian settings}

The key limitations and challenges we experienced during the eDEWS development and implementation included lack of pre-disaster preparedness, funding constraints, user retention, limited or no users technical awareness, human resource, accessibility to telecom networks, issues related to infrastructure/communication and IT, power availability, mobile phone subscriptions, delays in logistics, lack of motivation due to incentive based culture for added roles/responsibilities, and challenges in training delivery and follow-up at the district and health facility level.

It may take from 15 to 30 days to set up eDEWS surveillance system due to mandatory time required for environment scanning such as rapid assessment of IT infrastructure/communication and accessibility, resource mapping and needs assessment, requirements analysis, and finalizing logistics including local physical server that all are crucial steps to be considered before implementing any electronic tool for EWARN. These steps ensure higher success of project compared to projects implemented without understanding health informatics environment that involve people, process and technology. Moreover, such planning results in higher acceptance rate among end users, stake holders and government with sense of ownership due to local physical server since data resides within country and not shared on third country cloud server, a main concern raised by multiple government officials during eDEWS implementations in emergencies. This also encourage governments to switch and integrate eDEWS application as a part of WHO's recommended EWARN exit strategy and integrate into their routine disease surveillance system as evident from eDEWS integration into national disease surveillance system in Yemen [18,21,22].

\section{Discussion}

This paper presents our efforts to conceptualize and develop standardized informatics tools for disease early warning and share experiences in the use of eDEWS as a potential solution for standardized electronic data collection and reporting procedures during emergencies. eDEWS has supported implementation of communicable disease surveillance and early warning systems in humanitarian settings of Pakistan, Yemen, Somalia, Liberia and adapted in Iraq. The successful implementation of eDEWS in these countries demonstrates its potential to be adapted as a standardized electronic tools and data collection procedure for easy deployment during emergencies. The eDEWS application has been upgraded and improved based on lessons learned, best practices and experiences from its implementation in various countries since its first development and testing during 2011 flood emergency in Pakistan. Furthermore, recent upgrades are released considering various emergency scenarios, geographical terrains, communication and technological barriers and other technical challenges in order to save time and resources. eDEWS has also demonstrated successfully that it was able to meet its objectives in all humanitarian settings of generating timely notifications, outbreak containment, needs for automated epidemiological analysis, reporting and visualization, complementing routine surveillance, enhancing EWARN and filling gaps of disease surveillance in settings with non-existing surveillance systems. Currently, eDEWS program is functional in Yemen as integrated part of national integrated surveillance system $[18,19,21,22]$. 
If eDEWS is considered for implementation or adaptation as a standardized tool for EWARN during emergencies, the full deployment of eDEWS takes two weeks to a month from the beginning of an emergency response. This time includes environmental scanning, assessment, resource mapping, requirements analysis, prioritization, logistics, trainings, configuration of online database and app for smart screen devices when cloud computing is not an option for government. These steps are extremely crucial to set up any electronic tool during the emergencies and provides better understanding of local context and considerations such as technical, financial, cultural and political aspects that may otherwise result in project acceptance issues, government ownership or underperforming electronic tools and system, not meeting the objectives of EWARN system during emergencies.

To understand such challenges, address them properly and gather all necessary support for the eDEWS success, initial environment scanning was a part of eDEWS planning phase before launch, and we recommend applying similar approach in other similar interventions for successful implementation of electronic surveillance in low- and middle-income countries, especially during emergencies. We successfully addressed technical considerations in all settings such as IT infrastructure issues with development of mobile applications package, capable of transmitting data electronically from any geographical location such as good coverage (both internet and GSM/SMS services available), poor coverage (no internet but SMS/GSM service available) and no coverage (no internet or GSM/SMS service). The costs of such services were very well controlled to keep data transmission cost-efficient. To address power issues, we provided power banks that could provide continuous power supply to keep electronic devices charged for data collection purpose. The only issue we faced with this option was logistics to supply batteries but was manageable. Another main issue in using electronic means was monthly subscription process of mobile phone and internet services but we managed it centrally with memorandum of understanding with cellular companies, maintaining a log trail, better monitoring and documentation for accountability in eDEWS that helped in enforcing fair use policy.

For Liberia, we have noted special challenges with data transmission in situations of complete reliance on phone and electronic transmission of data for disease early warning purpose particularly in hard to reach areas, following a large-scale Ebola outbreak with significant impact. For such situation, we used portable booster satellite solution that allows satellite data transmission using smart phones, much cheaper than existing standard satellite phone options for electronic data transmission, to overcome challenges with data transmission in the event when communication networks are inoperable. Provision of such boosters ensured timely data transmission for an alert detection and notification purpose, a critical function of EWARN disease surveillance, aligned with WHO's 'no regrets' policy [5]. This approach also helped in bringing better solution to replace concept of "runner" being used by various eHealth projects implementing electronic tool in humanitarian settings when all communication systems had been destroyed. In this runner concepts, staff assigned as "runner" physically visit each of the reporting centers daily, collect data in electronic devices and transmits data electronically by visiting the nearest network coverage area.

Our results indicate that DST is very efficient and handy analytical approach to monitor health situation without any delay during humanitarian crises where poor capacity and issues with access 
and resources always cause delays in the implementation of electronic tools for disease early warning system. Furthermore, this innovative approach can strengthen overall public health information management capacities and capabilities in resource poor settings during emergencies due to its flexibility and simplicity in order to overcome the challenges in absence of electronic automated analysis tools and with limited skilled human resource capacities. The DST was introduced to compliment eDEWS system to fill the gap period required to set up eDEWS from the onset of emergency and can be used in parallel to setting up of EWARN system immediately upon emergency onset, thus covering bridging this essential gap.

Development and setting up of eDEWS system used emergency response funds in various humanitarian settings. The system was implemented with limited funds and an objective to be deactivated with EWARN when routine surveillance system resumes its full functionality, otherwise eDEWS has full potential to be integrated into routine surveillance system in a manner that ensures government ownership and sustainability but requires continuous support for maintenance cost that is much lower than the first time setting up cost. Hence, the deactivation of eDEWS project was more significantly driven by the lack of funds for continuation and its need in future by government or health sector. On contrary, eDEWS implementation in Yemen has resulted in successful integration of eDEWS system with national surveillance system, where project was initially pilot tested with limited funds in four governorates in 2013 but later secured more funding for project continuation and scaled up to cover all Yemen's governorates. This rapid expansion of the sentinel sites in all Yemen's governorates amid security challenges and limited resources has been documented as a considerable achievement and resulted in integration of eDEWS with national surveillance system into one system, called 'electronic integrated disease early warning system' (eIDEWS). This integration of eDEWS into national surveillance system has been found very effective that ensures the speed and efficiency of data collection, analysis and public health response to outbreaks [18,21,22].

\section{Conclusion}

This study demonstrates the feasibility of using eDEWS as standardized electronic tool to enhance EWARN implementation and other similar public health interventions requiring time efficient and cost-effective electronic tools for 'data into action' purpose in global humanitarian settings and incorporate as part of a regular emergency preparation programs. Furthermore, the recent integration of eDEWS into national routine surveillance system by Yemen demonstrate the potential of eDEWS to be integrated into national surveillance program (eIDEWS) to ensure the speed and efficiency of data collection, analysis and public health response to outbreaks. Such integration to 'one national integrated surveillance and response system' can only be achieved successfully through high level determination, commitment, trust building, strong collaboration and working relationships between government and health sector.

\section{Limitations}

Lack of access to the adapted eDEWS electronic tools in Iraq precluded meaningful comparison between eDEWS adaptation in Iraq with eDEWS project implementation in other humanitarian settings. The presented data on adapted eDEWS for EWARN in Iraq is taken through email 
communications and contacting teams supported eDEWS efforts to enhance EWARN in Iraq, thus limited in table presented in this paper.

Currently, two slightly different naming approaches are in use for the same concept of WHO's early warning and response (EWAR) during crisis in various humanitarian settings across the globe. These names are early warning and response system (EWARS) and early warning and response network (EWARN). To keep consistency across paper, we have used EWARN name, but the concept is same as EWARS and should not be confused.

\section{Acknowledgements}

We are grateful to WHO/EHA team leads, ministries of health, eDEWS teams, EWARN/DEWS teams, FELTP/CDC colleagues, WHO staff in EMRO/AFRO/HQ and country offices of Pakistan, Yemen, Somalia and Liberia, too numerous to list individually, who provided us with prompt support, information and facilitation for the concept development, designing, pilot testing, successful implementation and maintenance of eDEWS projects during crisis situations. Finally, we extend our thanks to all donors who funded the eDEWS project in all four countries.

\section{References}

1. Watson JT, Gayer M, Connolly MA. 2007. Epidemics after natural disasters. Emerg Infect Dis. 13(1), 1-5. PubMed https://doi.org/10.3201/eid1301.060779

2. Jones KE, Patel NG, Levy MA, Storeygard A, Balk D, et al. 2008. Global trends in emerging infectious diseases. Nature. 451(7181), 990-U994. PubMed https://doi.org/10.1038/nature06536

3. World Health Organization. 2000. An integrated approach to communicable disease surveillance. Available at: http://iris.paho.org/xmlui/bitstream/123456789/31907/1/EB_v21n1.pdf

4. World Health Organization. 2012. Outbreak surveillance and response in humanitarian emergencies: WHO guidelines for EWARN implementation. Available at: http://whqlibdoc.who.int/hq/2012/WHO_HSE_GAR_DCE_2012_1_eng.pdf?ua=1

5. World Health Organization. 2017. Emergency Response Framework (ERF). Available at: http://apps.who.int/iris/bitstream/10665/258604/1/9789241512299-eng.pdf?ua=1

6. World Health Organization. 2011. Early warning surveillance and response in emergencies. Available at: http://whqlibdoc.who.int/hq/2011/WHO_HSE_GAR_DCE_2011.2_eng.pdf?ua=1

7. Rahim M, Kazi BM, Bile KM, Munir M, Khan AR. 2010. The impact of the disease early warning system in responding to natural disasters and conflict crises in Pakistan. East Mediterr Health J. 16 Suppl, S114-121. 
8. Carney TJ, Shea CM. 2017. Informatics Metrics and Measures for a Smart Public Health Systems Approach: Information Science Perspective. Comput Math Methods Med., 1452415. PubMed https://doi.org/10.1155/2017/1452415

9. Savel TG, Foldy S. 2012. The role of public health informatics in enhancing public health surveillance. MMWR Suppl. 61(3), 20-24. PubMed

10. _Consulting VW mHealth for development: the opportunity of mobile technology for healthcare in the developing world. Washington and Berkshire (UK): UN FoundationVodafone Foundation Partnership; 2009

11. Yang C, Yang J, Luo X, Gong P. 2009. Use of mobile phones in an emergency reporting system for infectious disease surveillance after the Sichuan earthquake in China. Bull World Health Organ. 87(8), 619-23. PubMed https://doi.org/10.2471/BLT.08.060905

12. Robertson C, Sawford K, Daniel SLA, Nelson TA, Stephen C. 2010. Mobile Phone-based Infectious Disease Surveillance System, Sri Lanka. Emerg Infect Dis. 16(10), 1524-31. PubMed https://doi.org/10.3201/eid1610.100249

13. Ahmed K, Altaf MD, Dureab F. 2014. Electronic Infectious Disease Surveillance System during Humanitarian Crises in Yemen. ISSN 1947-2579. http://ojphi.org. Online J Public Health Inform. 6(1), e134.

14. World Health Organization. 2018. Public Health Information Services (PHIS) Toolkit. Available at: https://www.who.int/health-cluster/resources/publications/PHIS-toolkitOctober-2018.pdf?ua=1

15. World Health Organization. EWARNS dashboard in Iraq. 2015 [cited 2019 June 26]; Available from: http://www.emro.who.int/irq/ewarns/ewars-dashboard.html.

16. Ahmed K, Ajanga A, Saleh OA, Altaf MD, Popal GR. 2015. Application of an Innovative Data Analytical Approach to Assessing the Disease Situation during Crises in Somalia, Yemen and Pakistan. ISSN 1947-2579. http://ojphi.org. Online J Public Health Inform. 7(1), e101.

17. World Health Organization. Liberia introduces electronic reporting to detect disease outbreaks early. 2015 [cited 2019 June 26]; Available from:

https://www.afro.who.int/news/liberia-introduces-electronic-reporting-detect-diseaseoutbreaks-early

18. Mayad M, Alyusfi R, Assabri A, Khader Y. 2019. Evaluation of electronic Disease Early Warning System (eDEWS) in Sana'a governorate, Yemen. JMIR Preprints. [cited 2019 June 26]; Available from: https://preprints.jmir.org/preprint/14295 
19. Greenhalgh T, Peacock R. 2005. Effectiveness and efficiency of search methods in systematic reviews of complex evidence: audit of primary sources. BMJ. 331(7524), $1064-$ 65. PubMed https://doi.org/10.1136/bmj.38636.593461.68

20. World Health Organization. 2011. Evaluation of the Disease Early Warning System in crisisaffected areas of Pakistan. Available at:

https://www.who.int/hac/crises/pak/pakistan_dews_evaluation_report_mar2011.pdf

21. Dureab F, Ismail O, Muller O, Jahn A. 2019. Cholera Outbreak in Yemen: Timeliness of Reporting and Response in the National Electronic Disease Early Warning System. Acta Inform Med. 27(2), 85. PubMed https://doi.org/10.5455/aim.2019.27.85-88

22. World Health Organization. Electronic integrated disease early warning system launched in Yemen. 2015 [cited 2019 June 26]; Available from: http://www.emro.who.int/yem/yemennews/electronic-integrated-disease-early-warning-system-launched-in-yemen.html 